TRANSACTIONS OF THE

AMERICAN MATHEMATICAL SOCIETY

Volume 357, Number 9, Pages 3443-3458

S 0002-9947(04)03545-7

Article electronically published on May 10, 2004

\title{
RHOMBIC EMBEDDINGS OF PLANAR QUAD-GRAPHS
}

\author{
RICHARD KENYON AND JEAN-MARC SCHLENKER
}

\begin{abstract}
Given a finite or infinite planar graph all of whose faces have degree 4 , we study embeddings in the plane in which all edges have length 1 , that is, in which every face is a rhombus. We give a necessary and sufficient condition for the existence of such an embedding, as well as a description of the set of all such embeddings.

RÉsumé. Etant donné un graphe planaire, fini ou infini, dont toutes les faces sont de degré 4 , on étudie ses plongements dans le plan dont toutes les arêtes sont de longueur 1, c'est à dire dont toutes les faces sont des losanges. On donne une condition nécessaire et suffisante pour l'existence d'un tel plongement, et on décrit l'ensemble de ces plongements.
\end{abstract}

\section{INTRODUCTION}

Definition 1.1. Given a planar graph $G$ all of whose faces (except possibly for the outer face if $G$ is finite) have degree 4, called a quad-graph, a rhombic embedding is an embedding of $G$ in $\mathbb{R}^{2}$ with the property that all edges are line segments and have length 1 (and hence each bounded face is a rhombus).

Such embedding arises in discrete complex analysis [1, [5] and in statistical mechanics [3], 6], 4]. Here we study the spaces of such embeddings.

Our main results are the following:

(1) We give a simple necessary and sufficient condition (Theorem 3.1) for a planar graph to have a rhombic embedding in $\mathbb{R}^{2}$.

(2) We show that the space of rhombic embeddings of an infinite graph (with no unbounded faces) is a convex set when parametrized by the rhombus angles. There is a simple description of the extreme points of the closure of this convex set. (Section 4.1 and Theorem 4.10.)

(3) We consider the space of periodic rhombic embeddings of a periodic planar graph. It is the interior of a convex polyhedron. The area of the fundamental domain provides a strictly convex functional on this polyhedron; we give a geometric description of the unique critical point (Theorem [5.2).

Instead of rhombic embeddings, one can consider the more general notion of parallelogram embeddings, in which each face is mapped to a parallelogram. Some of the results of this paper extend to parallelogram embeddings; this is explained in section 6. Parallelogram embeddings arise in the study of discrete non-linear integrable models and discrete conformal maps (see [2]).

Received by the editors June 18, 2003 and, in revised form, September 15, 2003.

2000 Mathematics Subject Classification. Primary 52Cxx.

(C)2004 American Mathematical Society 


\section{BACKGROUND AND MOTIVATIONS}

2.1. Discrete complex analysis and statistical mechanics. Rhombic embeddings first appeared in Duffin [1, in the context of discrete complex analysis. Indeed, as Duffin shows, there is a natural way to define discrete analytic functions on graphs with rhombic embeddings, which do not appear to generalize to arbitrary embeddings.

Duffin's ideas were rediscovered by Mercat [6], [5] who used them to build up an extended theory of discrete holomorphy in one complex dimension.

In [3], the closely related concept of isoradial embeddings (see the next section) was shown to be useful in the study of the so-called dimer model of statistical mechanics. By choosing edge interactions to be a particular function of the corresponding edge lengths in the isoradial embedding, certain simplifications (commutation relations) appear which do not appear for more general energies. These allow one to give explicit solutions to correlation functions in the dimer model on isoradial graphs.

Other statistical mechanical models such as the Ising model and more generally the random cluster model also become simpler on isoradial graphs [6], [4]. In fact, even the simple random walk behaves nicely, as is evidenced by an explicit formula for the Green's function [3].

2.2. Isoradial embeddings. An isoradial embedding (see [3]) of a planar graph $\Gamma$ is a locally finite embedding in $\mathbb{R}^{2}$ with the property that each bounded face $f$ is a cyclic polygon (inscribable in a circle) with circumcircle of radius 1 . The center of the circumcircle $C$ is also called the center of $f$. The embedding is said to be convex if the center of a face is contained in the closure of the face. It is strictly convex if the center is in the interior of the face.

Given a planar graph $\Gamma$, the diamond graph $G$ associated to $\Gamma$ is the graph whose vertices are the union of the vertices and faces of $\Gamma$, and with an edge between each face and vertex on that face. The faces of $G$ are the edges of $\Gamma$. The faces of $G$ are of degree 4 .

It is clear that a strictly convex isoradial embedding of a graph $\Gamma$ gives a rhombic embedding of $G$. Conversely, a rhombic embedding of $G$ defines a strictly convex isoradial embedding of $\Gamma$. Furthermore every quad-graph arises as the diamond graph not only of the graph $\Gamma$, but also of its Poincaré dual $\Gamma^{\prime}$, where the faces of one graph correspond to the vertices of the other. So the study of strictly convex isoradial embeddings and rhombic embeddings is equivalent. Therefore, we will work only with the quad-graph $G$.

There is an underlying relation between isoradial embeddings, in particular the periodic ones, and hyperbolic geometry. This is particularly apparent in [3]. Those relations are however not of primary importance for the aspects which we have considered here, so we will not mention them further.

\section{Rhombic EMBeddings}

In this paper a planar graph will mean a graph with a preferred isotopy class of embeddings in the plane. An embedding of such a graph will always mean an embedding in the same isotopy class.

We will only deal with either finite graphs, all of whose faces except the outer face have degree 4 , or with infinite graphs with no unbounded faces, that is, all 
of whose faces have degree 4. In either case we refer to such graphs as planar quad-graphs, with the understanding that we exclude the outer face from this restriction in the case of a finite graph. Although the results in this section apply in greater generality, for simplicity we deal only with these cases.

In a planar quad-graph $G$, a train-track is a path of faces (each face being adjacent along an edge to the previous face) which does not turn: on entering a face it exits across the opposite edge. We assume that the train-tracks extend in both directions as far as possible, that is, they are not embedded in a longer train-track. This means they are either periodic, or extend infinitely far in both directions, or extend until they enter the outer face of $G$ if $G$ is finite. $\operatorname{Let} \operatorname{Tr}(G)$ denote the set of train-tracks of $G$. In a rhombic embedding, each rhombus in a train-track has an edge parallel to a fixed unit vector $u$. In an oriented train-track we choose the direction of $u$ so that when the train runs down, the track $u$ points from the right to the left. The vector $u$ is called the transversal of the oriented train-track. Those notions should be easily identified in figure 1 which was made following a suggestion of the referee.

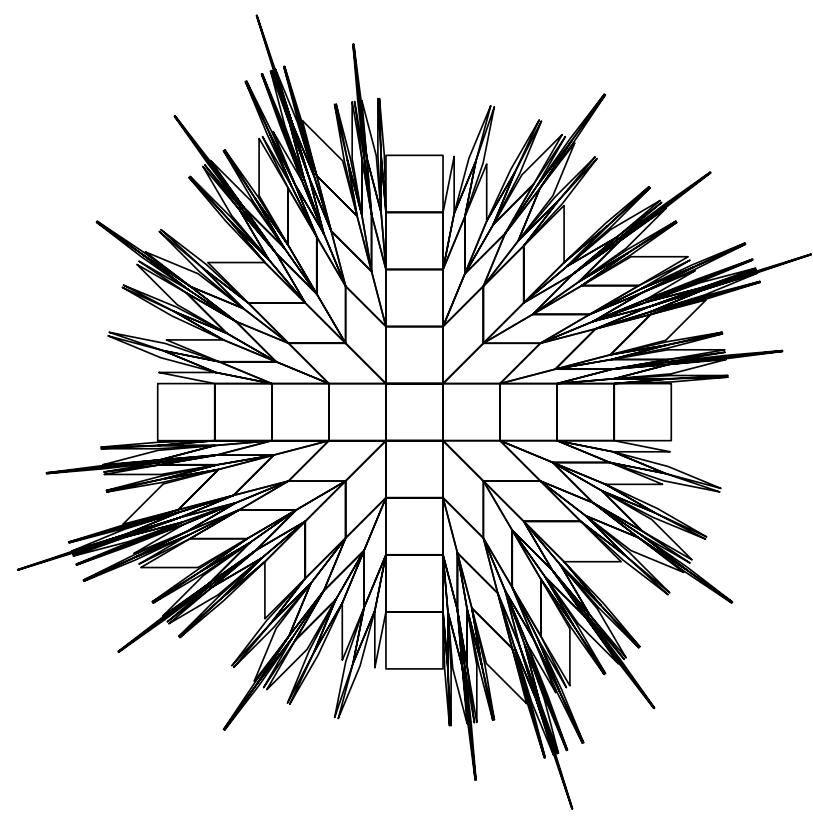

FiguRE 1. Rhombic embedding of the infinite planar graph with faces of degree 4 and vertices of degree 5 .

Theorem 3.1. A planar quad-graph $G$ has a rhombic embedding in the plane if and only if the following two conditions are satisfied:

(1) No train-track path crosses itself or is periodic.

(2) Two distinct train-tracks cross each other at most once.

The conditions are clearly necessary: in a rhombic embedding each train-track is a monotone path (in the direction perpendicular to its common parallel). Therefore a train-track cannot cross itself. If two oriented train-tracks with transversals $u$ and 
$v$ cross (with the first crossing from right to the left of the second), the rhombus on which they cross has edges $u$ and $v$ and $u \wedge v>0$. If they crossed again the first would cross from the left to the right of the second and the rhombus would have the same two edges but in the reverse orientation, that is, it would have negative area.

It remains to construct a rhombic embedding for any graph satisfying the conditions. Enumerate the distinct train-tracks $\left\{t_{1}, t_{2}, \ldots\right\}$. Let $G_{k}$ be the union of the faces contained in the first $k$ train-tracks $\left\{t_{1}, \ldots, t_{k}\right\}$. The $G_{k}$ are subgraphs of $G$ and $G=\bigcup_{k=1}^{\infty} G_{k}$. Similarly let $G_{k}^{\prime}$ be the subgraph of the 4 -valent dual $G^{\prime}$ which is the union of its edge paths comprising the first $k$ train-tracks.

Lemma 3.2. There exists a topological embedding $\rho$ of $G^{\prime}$ in the unit disk $\mathbb{D}$, such that the image of each path of $G^{\prime}$ (which corresponds to a train-track in $G$ ) is a smooth path connecting distinct boundary points of the disk.

Proof. Since $G^{\prime}$ is planar, first choose a topological embedding of $G^{\prime}$ in the disk. For each $k$, by removing all but the first $k$ train-tracks from this embedding, we get topological embeddings of $G_{1}^{\prime}, G_{2}^{\prime}, \ldots$, in which the embedding of $G_{k}^{\prime}$ is an extension of that of $G_{k-1}^{\prime}$.

To make a sequence of smooth embeddings, with appropriate boundary behavior of the train-tracks, proceed inductively as follows. Choose any non-trivial chord for $G_{1}^{\prime}$. Suppose now that the smooth embedding of $G_{k}^{\prime}$ has been defined, isotopic to the original topological embedding. The graph $G_{k+1}^{\prime}$ is obtained from $G_{k}^{\prime}$ by adding a single line $t_{k+1}$ which has a finite number of intersections with the lines present in $G_{k}^{\prime}$. On top of the smooth embedding of $G_{k}^{\prime}$ draw in the train-track $t_{k+1}$ as a smooth path, respecting its intersections (and their order) with each of the $t_{j}$ for $j \leq k$, that is, so that the resulting embedding of $G_{k}^{\prime} \cup t_{k+1}=G_{k+1}^{\prime}$ is topologically equivalent to the original topological embedding of $G_{k+1}^{\prime}$. Since $t_{k+1}$ has only a finite number of intersections with $G_{k}^{\prime}$, it can be chosen so that its endpoints are distinct points on the boundary of the disk. This defines the smooth embedding of $G_{k+1}^{\prime}$. The union of these embeddings over all $k$ is a planar embedding of $G^{\prime}$ : the image of any finite piece of $G^{\prime}$ is fixed after a finite number of steps of this algorithm.

To each oriented train-track $t_{j}$ we associate the unit vector $u_{j}$ which is perpendicular to the chord joining its two endpoints in the above embedding. We construct the rhombic embedding of $G$ as follows. Each face of $G$ is crossed by exactly two train-tracks, $t_{j}$ and $t_{k}$. To this face associate the rhombus with edges $u_{j}$ and $u_{k}$. Its angles are positive. Glue two rhombi together along an edge if they are adjacent faces in $G$; this defines a simply connected locally Euclidean surface. It remains to show that it is isometric to a subset of the plane.

This surface has a natural projection $\phi$ to the plane, which clearly is locally injective. We have to prove that $\phi$ is injective.

Lemma 3.3. Let $v, w$ be two vertices of $G$, and let $t_{k_{1}}, \cdots, t_{k_{n}}$ be the train-tracks separating $v$ and $w$; assume these tracks are oriented so that $v$ is on the right and $w$ is on the left. Then

$$
\phi(w)-\phi(v)=\sum_{j=1}^{n} u_{k_{j}} .
$$


Proof. Let $\Gamma$ be any path going from $v$ to $w$ in the 1-skeleton of $G$. $\Gamma$ is a sequence of edges of $G$, each corresponding to a train-track. Each of the train-tracks $t_{k_{1}}, \cdots, t_{k_{n}}$ appears an odd number of times, and is crossed one more time from right to left than from left to right. All the other train-tracks are crossed the same number of times in both directions. So the total translation vector of $\Gamma$ is a sum of terms which pairwise cancel, except those in (3.1).

Let $X$ and $Y$ be two distinct faces of $G$. We will show that their $\phi$-images have disjoint interiors. $X$ and $Y$ correspond to vertices $x$ and $y$, respectively, in the graph $G^{\prime}$. We choose $k$ so large that the train-tracks containing $X$ and $Y$, as well as the train-tracks which go between $X$ and $Y$, are in $\left\{t_{1}, \cdots, t_{k}\right\}$.

Lemma 3.4. There exists an embedded path $\gamma:[0,1] \rightarrow \mathbb{D}^{2}$, with endpoints on the boundary of the disk, which goes through $\rho(x)$ and $\rho(y)$, and crosses each $\rho\left(t_{j}\right), 1 \leq$ $j \leq k$, at most once.

Proof. Consider the restriction of the embedding $\rho$ to $G_{k}^{\prime}$. We continuously deform it so that the path corresponding to each $t_{j}$ becomes a chord of the circle with the same endpoints. Under this deformation some topological changes may take place: a path may move past an intersection of two other paths as in Figure 2 As a result the deformed graph $\tilde{G}_{k}^{\prime}$ is not necessarily isomorphic to $G_{k}^{\prime}$, but can be obtained from $G_{k}^{\prime}$ by a finite sequence of these triple-crossing moves. Moreover, there is a natural bijection between the vertices of $\tilde{G}_{k}^{\prime}$ and those of $G_{k}^{\prime}$. In $\tilde{G}_{k}^{\prime}$ draw $\gamma$ as a chord passing through $\rho(x)$ and $\rho(y)$. By a general position argument we can assume that no three chords meet at a point, and $\gamma$ does not meet any other chord intersections. Now undo the deformation, passing from $\tilde{G}_{k}^{\prime} \cup \gamma$ to $G_{k}^{\prime}$, undoing the triple-crossing moves in sequence. It is clear how to deform $\gamma$ at the same time, keeping the property of $\gamma$ passing through $\rho(x)$ and $\rho(y)$, so that $\gamma$ never crosses any path more than once; see Figure 3 which shows how $\gamma$ may be deformed at each triple crossing. This completes the construction of $\gamma$.

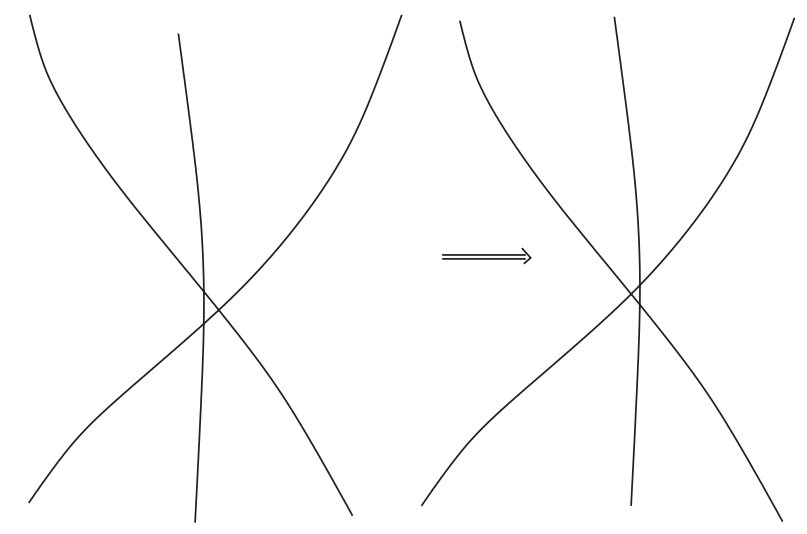

FIGURE 2. Moving a strand past an intersection.

The fact that $X$ and $Y$ have disjoint images, and therefore the proof of Theorem 3.1, follows from the next lemma. 

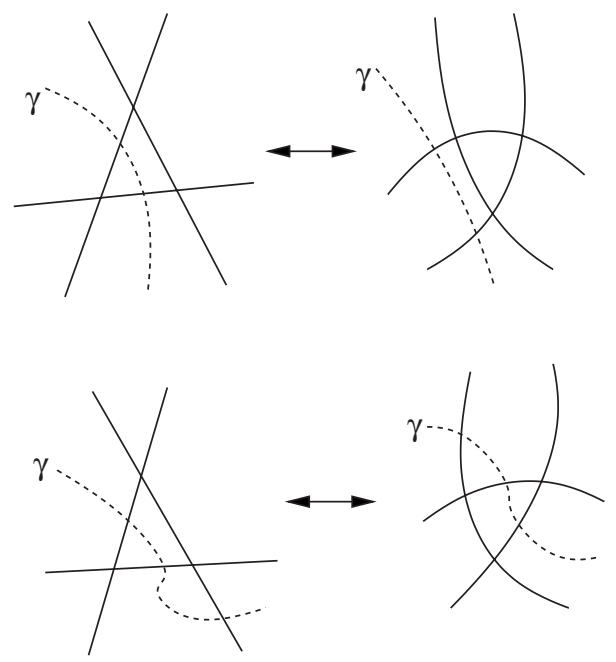

Figure 3. Moving $\gamma$ at a triple intersection.

Lemma 3.5. Let $v$ and $w$ be vertices of $X$ and $Y$, respectively. The orthogonal projection of $\phi(w)-\phi(v)$ on the oriented line $(\gamma(0) \gamma(1))$ has positive orientation.

Proof. Let $t_{k_{1}}, \cdots, t_{k_{n}}$ be the oriented train-tracks which have $v$ on their right and $w$ on their left. By the choice of $k$ above, $t_{k_{j}} \leq k$ for all $j \in\{1, \cdots, n\}$. By Lemma 3.3 .

$$
\phi(w)-\phi(v)=\sum_{j=1}^{n} u_{k_{j}} .
$$

But, for all $j \in\{1, \cdots, n\}, t_{k_{j}}$ intersects $\gamma$, so it has $\gamma(0)$ on its right and $\gamma(1)$ on its left. By construction of $u_{k_{j}}$, it implies that the orthogonal projection on $(\gamma(0) \gamma(1))$ of $u_{k_{j}}$ is positively oriented, and the result follows.

\section{The SPACE OF RHOMBIC EMBedDings}

In this section we suppose that $G$ is infinite. We will study the space of rhombic embeddings of $G$, and show that its closure is parametrized by $F_{\geq}\left(\operatorname{Tr}(G), S^{1}\right)$, a quotient of a space of maps from the train-tracks of $G$ to the circle, forming a convex polytope (of infinite dimension). We will describe its vertices, which are ways to flatten the graph on the line $\mathbb{Z}$. We will then consider the periodic case.

4.1. Linear parametrization. A wedge in a planar graph is a pair consisting of a face and a vertex on that face. We let $W$ denote the set of wedges of a quad-graph $G$.

A rhombic embedding determines an angle in $(0, \pi)$ for each wedge of $G$, satisfying some simple linear conditions. The converse is also true.

Theorem 4.1. Let $\alpha: W \rightarrow(0, \pi)$ be a map such that:

- adjacent wedges on the same face have angles summing to $\pi$.

- the sum of the angles around each vertex is $2 \pi$.

Then $\alpha$ is obtained on a unique rhombic embedding $\phi$ of $G$ in $\mathbb{R}^{2}$, up to an isometry. The image of $\phi$ is either a plane, a half-plane, or an infinite strip. 
Note that $\phi(G)$ can indeed be a half-plane or a strip. it is easy to find examples of this when $G=\mathbb{Z}^{2}$ (see Figure 4 ). The proof of this theorem will be given after the next lemmas.

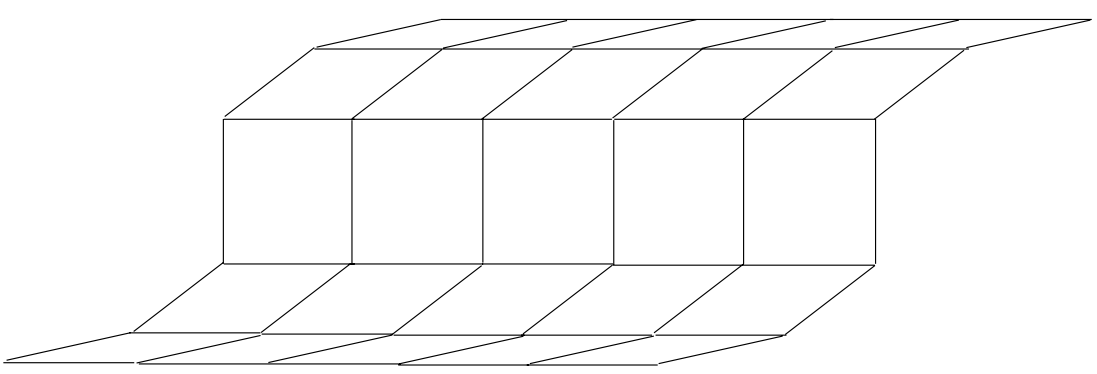

FiguRE 4. An embedding of $\mathbb{Z}^{2}$ with image a strip.

Lemma 4.2. Let $G_{0}$ be a finite quad-graph. Let $v_{0}$ be a vertex of $G_{0}$ which is at (combinatorial) distance $k$ from the outer face. For each $\epsilon>0$, there exists $\alpha>0$ such that, if $\phi$ is a rhombic immersion of $G_{0}$ in $\mathbb{R}^{2}$ and each face has area at most $\alpha$, then:

- $\phi\left(G_{0}\right)$ is $\epsilon$-close to a line $L$ containing $\phi\left(v_{0}\right)$.

- there exists a segment of $L$ of length $2 k$, centered at $\phi\left(v_{0}\right)$, which is $\epsilon$-close to $\phi\left(G_{0}\right)$.

Proof. $\phi$ is uniquely determined (up to translation) by the transverse directions of the train-tracks in $G_{0}$. Moreover, if two intersecting train-tracks have transverse directions which are neither close nor almost opposite, the area of the faces cannot all be small. Therefore, the transverse directions of all train-tracks in $G_{0}$ are close to either $u$ or to $-u$, for some $u \in S^{1}$.

This already shows that $\phi\left(G_{0}\right)$ remains close to the line containing $\phi\left(x_{0}\right)$ and of direction $u$. In addition, if $v$ is a vertex of $G_{0}$ which is not adjacent to the outer face, then the edges starting from $v$ cannot all be pointing in directions close to $u$ or all in the directions close to $-u$-there must be some pointing in both directions. Since $v_{0}$ is at distance at least $k$ from the outer face, the segment of length $2 k$ centered at $\phi\left(v_{0}\right)$ and directed by $u$ is close to $\phi\left(G_{0}\right)$.

Lemma 4.3. Let $\left(\phi_{t}\right)_{t \in[0,1]}$ be a 1-parameter family of rhombic immersions of $G$ (i.e. not necessarily globally injective). Suppose that, for all $t \in[0,1), \phi_{t}$ is an embedding. Then $\phi_{1}$ is an embedding, and $\phi_{1}(G)$ is either a plane, a half-plane or a strip.

Proof. Define a boundary point of $\phi_{1}$ as a point $x \in \mathbb{R}^{2}$ such that any neighborhood of $x$ intersects an infinite set of rhombi of $\phi(G)$. If $\phi_{1}$ has no boundary point, then it is a local homeomorphism and it is proper, so that it is a global homeomorphism; thus it is an embedding, with image $\mathbb{R}^{2}$. So we suppose that $\phi_{1}$ has a boundary point $x_{0}$.

Since the $\phi_{t}$ are embeddings for $t<1$, for any $k \in \mathbb{N}$, the sum of the areas of the rhombi of $\phi_{1}(G)$ which are within Euclidean distance at most $k$ from $x_{0}$ is 
finite (bounded by the area of the disk of radius $k+1$ ). However, by definition of a boundary point, there is a sequence of vertices $v_{n}$ of $G, n \in \mathbb{N}$, such that $\left(\phi_{1}\left(v_{n}\right)\right) \rightarrow x_{0}$ and that the disks $D_{n}$ of (combinatorial) radius $k$ centered on the $v_{n}$ are disjoint in $G$. Then the area of the $\phi_{1}\left(D_{n}\right)$ go to 0 as $n \rightarrow \infty$.

By the lemma above, for $n$ large enough, the $\phi_{1}\left(D_{n}\right)$ are each close to a line going through $x_{0}$. By construction of $t_{0}$, they are disjoint, so they are all close to a fixed line $L \ni x_{0}$. Moreover, still by the lemma, there is a segment $\sigma$ of $L$ of length $2 k$ centered at $x_{0}$ which is arbitrarily close to the $\phi_{1}\left(D_{n}\right)$ for $n$ large enough.

Therefore, all points of $\sigma$ are boundary points of $\phi_{1}$. This is true for all $k \in \mathbb{N}$, so the line $L$ is completely made up of boundary points of $\phi_{1}$. What is more, $\phi_{1}(G)$ cannot be on both sides of $L$, since otherwise $G$ could not be connected. Since the same can be said of all the boundary points of $\phi_{1}$, we have that $\phi_{1}(G)$ is bounded by disjoint lines. There can be either one or two of those lines; in the first case $\phi_{1}(G)$ is a half-plane, in the second case it is a strip. This description shows that $\phi_{1}$ is globally injective, so it is an embedding.

It is now possible to prove Theorem 4.1

Proof. Given a set of angles satisfying these two linear conditions, there corresponds at most one rhombic embedding up to global isometry. Conversely, it is clear that such a choice of angles determines a locally injective map from $G$ to $\mathbb{R}^{2}$, but it remains to show that it is globally injective.

The space of maps $\alpha: W \rightarrow(0, \pi)$ satisfying the two linear conditions is convex. We know from Theorem 3.1 that there exists a choice of angles, say $\alpha_{0}$, giving an embedded graph. We call $\left(\alpha_{t}\right)_{t \in[0,1]}$ the affine parametrized segment of maps $\alpha_{t}: W \rightarrow(0, \pi)$ between $\alpha_{0}$ and $\alpha_{1}=\alpha$, and $\left(\phi_{t}\right)_{t \in[0,1]}$ the associated 1-parameter family of rhombic immersions of $G$.

We will show that $\phi_{t}$ is an embedding - i.e. is globally injective - for all $t \in[0,1)$. Lemma 4.3 then shows that $\phi_{1}$ is an embedding, with image a plane, half-plane or strip. Suppose $\phi_{t}$ is not an embedding for all $t$; let $t_{0}$ be the infimum of the $t \in[0,1]$ such that $\phi_{t}$ is not globally injective, and we suppose that $t_{0}<1$.

Lemma 4.3, applied to the 1-parameter family $\left(\phi_{t}\right)_{t \in\left[0, t_{0}\right]}$, shows that $\phi_{t_{0}}$ is an embedding, and that its image is either a plane, a half-plane or a strip. Moreover, the definition of $\left(\phi_{t}\right)$ from the affine segment $\left(\alpha_{t}\right)_{t \in[0,1]}$ shows that no rhombus angle goes to 0 as $t \rightarrow t_{0}$. Therefore $\phi_{t_{0}}$ cannot have boundary points, and its image is a plane. For $t \geq t_{0}$ close enough to $t_{0}$, each of the rhombus angles remains bounded from below, so $\phi_{t}$ is still proper, and thus an embedding. This contradicts the definition of $t_{0}$. So $\phi_{t}$ is an embedding for all $t \in[0,1]$.

4.2. Convexity. Theorem 4.1 proves that the space of rhombic embeddings is parametrized by the choice of an angle for each wedge, satisfying the above linear conditions. Thus the space of rhombic embeddings is the intersection of a cube $(0, \pi)^{W}$ with the set of linear subspaces defined by these constraints. Thus it is a convex set, which we denote $\operatorname{Rh}(G)$.

Given a quad-graph $G$, we will call $\operatorname{Tr}_{+}(G)$ the set of oriented train-tracks. So $\operatorname{Tr}_{+}(G)$ has two elements for each train-track $t \in \operatorname{Tr}(G)$, one for each choice of orientation of $t$. For each $t \in \operatorname{Tr}_{+}(G)$, we call $-t$ the oriented train-track which is the same as $t$ but with the opposite orientation.

We consider now only graphs which satisfy the hypothesis of Theorem 3.1. In particular, two oriented train-tracks $t, t^{\prime}$ with $t \neq \pm t^{\prime}$ intersect at most once. Given 
two distinct oriented train-tracks $t, t^{\prime} \in \operatorname{Tr}_{+}(G)$, with $t^{\prime} \neq \pm t$, we will say that $t^{\prime}$ has positive intersection with $t$ if $t$ and $t^{\prime}$ intersect, and the intersection has positive orientation, that is, if $t^{\prime}$ crosses $t$ from right to left.

Let $\mathcal{D}$ be the directed graph whose vertices are the oriented train-tracks, with an edge going from $t$ to $t^{\prime}$ if and only if $t^{\prime}$ intersects $t$ positively. In particular, if $t^{\prime}$ intersects $t$ positively, then $\mathcal{D}$ has a cycle $t \rightarrow t^{\prime} \rightarrow-t \rightarrow-t^{\prime} \rightarrow t$.

Let $\phi \in \operatorname{Rh}(G)$ be a rhombic embedding of $G$. Then each oriented train-track has a transversal, which is a unit vector, i.e. an element of $S^{1}$. Thus $\phi$ induces a map $\operatorname{Tr}_{+}(G) \rightarrow S^{1}$, with the property that $\phi(-t)=\phi(t)+\pi$. We will see below that this map is strictly order-preserving in the following sense.

Definition 4.4. (1) We call $F\left(\operatorname{Tr}(G), S^{1}\right)$ the space of maps $\theta$ from $\operatorname{Tr}_{+}(G)$ to $S^{1}$ such that, for all $t \in \operatorname{Tr}_{+}(G), \theta(-t)=\theta(t)+\pi$.

(2) A map $\theta \in F\left(\operatorname{Tr}(G), S^{1}\right)$ is strictly order preserving if, for each $t, t^{\prime} \in$ $\operatorname{Tr}_{+}(G)$ such that $t^{\prime}$ has positive intersection with $t, \theta\left(t^{\prime}\right) \in(\theta(t), \theta(t)+\pi)$. We call $F_{>}\left(\operatorname{Tr}(G), S^{1}\right)$ the space of strictly order-preserving elements of $F\left(\operatorname{Tr}(G), S^{1}\right)$.

(3) A map $\theta \in F\left(\operatorname{Tr}(G), S^{1}\right)$ is order-preserving if:

- For each $t, t^{\prime} \in \operatorname{Tr}_{+}(G)$ such that $t^{\prime}$ intersects $t$ positively, $\theta\left(t^{\prime}\right) \in$ $[\theta(t), \theta(t)+\pi]$.

- Each subgraph of $\mathcal{D}$ consisting of train-tracks having a fixed $\theta$-value is acyclic (has no oriented cycles).

We call $F_{\geq}\left(\operatorname{Tr}(G), S^{1}\right)$ the space of order-preserving elements of $F\left(\operatorname{Tr}(G), S^{\overline{1}}\right)$.

Lemma 4.5. A map $\theta \in F\left(\operatorname{Tr}(G), S^{1}\right)$ determines a rhombic embedding of $G$ if and only if it is strictly order-preserving.

Proof. Given a rhombic embedding of $\phi$ of $G$, we have already mentioned that it induces a map $u \in F\left(\operatorname{Tr}(G), S^{1}\right)$. If two oriented train-tracks $t, t^{\prime} \in \operatorname{Tr}_{+}(G)$ intersect, then the intersection rhombus has positive orientation and this means that, if $t^{\prime}$ has positive intersection with $t$, then $u\left(t^{\prime}\right) \in(u(t), u(t)+\pi)$. Thus $u$ is strictly order-preserving.

Conversely, let $u \in F_{>}\left(\operatorname{Tr}(G), S^{1}\right)$. Let $w=(f, v)$ be a wedge in $G$, where $f$ is a face and $v$ is a vertex of $f$. Let $t_{1}$ and $t_{2}$ be the two oriented train-tracks such that the oriented edges $e_{1}$ and $e_{2}$ of $f$ starting from $v$ are the oriented transverse directions of $t_{1}$ and $t_{2}$, respectively. Define a map $\alpha: W \rightarrow \mathbb{R}$ by setting $\alpha(w)$ equal to the oriented angle between $u\left(t_{1}\right)$ and $u\left(t_{2}\right)$. Since $u$ is strictly order-preserving, $\alpha$ takes its values in $(0, \pi)$. It is clear that $\alpha$ satisfies the two linear conditions in Theorem 4.1 .

Thus Theorem 4.1 shows that $\alpha: W \rightarrow(0, \pi)$ is obtained on a rhombic embedding of $G$, for which - up to an isometry in $\mathbb{R}^{2}$ — the transverse direction of each oriented train-track $t$ is given by $u(t)$.

Clearly two maps $\theta, \theta^{\prime} \in F_{>}\left(\operatorname{Tr}(G), S^{1}\right)$ determine the same rhombic embedding, up to a global isometry, if and only if there exists a constant $\theta_{0}$ such that, for all $t \in \operatorname{Tr}_{+}(G), \theta^{\prime}(t)=\theta(t)+\theta_{0}$. Therefore, $\operatorname{Rh}(G)$ is the quotient of $F_{>}\left(\operatorname{Tr}(G), S^{1}\right)$ by $S^{1}$.

Lemma 4.6. The closure of $F_{>}\left(\operatorname{Tr}(G), S^{1}\right)$ is $F_{\geq}\left(\operatorname{Tr}(G), S^{1}\right)$. 
Proof. Let $\theta \in F\left(\operatorname{Tr}(G), S^{1}\right)$. We want to show that the following statements are equivalent:

(1) $\theta$ is order-preserving.

(2) For all $\epsilon>0$, there exists $\theta^{\prime} \in F_{>}\left(\operatorname{Tr}(G), S^{1}\right)$ which is strictly orderpreserving and such that, for all $t \in \operatorname{Tr}_{+}(G),\left|\theta^{\prime}(t)-\theta(t)\right|<\epsilon$.

It is clear that (2) implies (1), so we only have to prove the converse. Let $\theta \in F\left(\operatorname{Tr}(G), S^{1}\right)$ be order-preserving.

We can partition the set of train-tracks into sets which are the maximal components of $\mathcal{D}$ having the same $\theta$-value. Without loss of generality we assume that the value of $\theta$ is different on each component. Enumerate the components $C_{1}, C_{2}, \ldots$; we can further assume by perturbing $\theta$ slightly (keeping the value constant on each $C_{i}$ ) that for $i<j$ the $\theta$ values of $C_{i}$ and $C_{j}$ are at least $\epsilon / 2^{j}$ apart.

We now perturb the $\theta$-values within each $C_{i}$ so that the new values lie in $\left(\theta-\epsilon / 2^{i+1}, \theta+\epsilon / 2^{i+1}\right)$. But $\mathcal{D}$ restricted to $C_{i}$ is an acyclic connected graph; as such it represents a partial order of its (at most countably many) vertices. Assign values in $\left(\theta-\epsilon / 2^{i}, \theta+\epsilon / 2^{i}\right)$ to the train-tracks in a way which is compatible with this (strict) partial order. This completes the construction.

Lemma 4.7. The natural map from $F_{>}\left(\operatorname{Tr}(G), S^{1}\right)$ to $\operatorname{Rh}(G)$ extends continously to a map from $F_{\geq}\left(\operatorname{Tr}(G), S^{1}\right)$ to the closure of $\operatorname{Rh}(G)$ (for its affine structure). The induced map from $F_{\geq}\left(\operatorname{Tr}(G), S^{1}\right) / S^{1}$ to $\overline{\operatorname{Rh}(G)}$ is one-to-one.

Proof. It is clear that the map from $F_{>}\left(\operatorname{Tr}(G), S^{1}\right)$ to $\operatorname{Rh}(G)$ extends continously to a map from $F_{\geq}\left(\operatorname{Tr}(G), S^{1}\right)$ to $\overline{\operatorname{Rh}(G)}$, which is surjective by construction.

Given $u \in F_{\geq}\left(\operatorname{Tr}(G), S^{1}\right), u$ is uniquely determined - up to an isometry in $\mathbb{R}^{2}$ - by the angles at the wedges, which are now in $[0, \pi]$, i.e. by the image of $u$ in $\overline{\operatorname{Rh}(G)}$. So the induced map from $F_{\geq}\left(\operatorname{Tr}(G), S^{1}\right) / S^{1}$ to $\overline{\operatorname{Rh}(G)}$ is injective.

As a consequence, the affine structure on $\operatorname{Rh}(G)$ can be obtained from the parametrization by the wedge angles, but also from the affine structure on $F_{>}\left(\operatorname{Tr}(G), S^{1}\right)$ through the quotient by $S^{1}$. This second description will be useful below to understand the extreme points of this convex set.

4.3. The group of circle homeomorphisms. A consequence of the definitions of $F\left(\operatorname{Tr}(G), S^{1}\right), F_{\geq}\left(\operatorname{Tr}(G), S^{1}\right)$ and $F_{>}\left(\operatorname{Tr}(G), S^{1}\right)$ is that, for each $G$, there is a canonical action on each of those convex sets of the group of homeomorphisms of the circle.

Definition 4.8. We call $\mathcal{H}$ the group of homeomorphisms $\phi: S^{1} \rightarrow S^{1}$ such that, for all $x \in S^{1}, \phi(-x)=-\phi(x)$.

There is a natural action of $\mathcal{H}$ on $F\left(\operatorname{Tr}(G), S^{1}\right)$, defined as follows. Let $\phi \in \mathcal{H}$. For each $\theta \in F\left(\operatorname{Tr}(G), S^{1}\right)$ and each $t \in \operatorname{Tr}_{+}(G)$, let $\phi^{*}(\theta)(t):=\phi(\theta(t))$. It is clear that this defines an action of $\mathcal{H}$ on $F\left(\operatorname{Tr}(G), S^{1}\right)$. Moreover, given $\phi \in \mathcal{H}$, it is also clear that an element $\theta \in F\left(\operatorname{Tr}(G), S^{1}\right)$ is (strictly) order-preserving if and only if $\phi(\theta)$ is (strictly) order-preserving, and therefore $\mathcal{H}$ also acts on $F_{\geq}\left(\operatorname{Tr}(G), S^{1}\right)$ and $F_{>}\left(\operatorname{Tr}(G), S^{1}\right)$. Note that the action of $\phi$ on $F\left(\operatorname{Tr}(G), S^{1}\right)$ is not affine.

By Lemmas 4.5 and 4.6, $F_{\geq}\left(\operatorname{Tr}(G), S^{1}\right)$ is a closed convex set. Thus it has a natural stratification, with one codimension 0 cell corresponding to $F_{>}\left(\operatorname{Tr}(G), S^{1}\right)$, and higher-dimensional stratums corresponding to some intersecting train-tracks having the same image in $S^{1}$. 
Lemma 4.9. The action of $\mathcal{H}$ preserves each stratum of $F_{\geq}\left(\operatorname{Tr}(G), S^{1}\right)$.

Proof. This is again a consequence of the definitions.

4.4. Vertices. The previous considerations lead to two interpretations of the extreme points of $\operatorname{Rh}(G)$, one in terms of maps from $\operatorname{Tr}_{+}(G)$ to $\{ \pm 1\}$, the other in terms of "flattenings" of the graph on a line.

Theorem 4.10. The extreme points of $\operatorname{Rh}(G)$ are in one-to-one correspondence with the maps $\theta: \operatorname{Tr}_{+}(G) \rightarrow\{-1,1\}$ satisfying $\theta(-t)=-\theta(t)$, such that the subgraphs $\mathcal{D}_{1}$ and $\mathcal{D}_{-1}$ of $\mathcal{D}$, consisting of vertices with values \pm 1 , respectively, are acyclic.

This theorem is a direct consequence (through the quotient by $S^{1}$ ) of the following lemma on the geometry of $F_{\geq}\left(\operatorname{Tr}(G), S^{1}\right)$. To state it, we introduce a simple notation. Let $T \subset \operatorname{Tr}_{+}(G)$ be a subset such that, for each $t \in \operatorname{Tr}_{+}(G)$, either $t \in T$ or $-t \in T$, but not both ( $T$ is a choice of orientation for each train-track). Then, for $\alpha \in S^{1}$, define

$$
\begin{aligned}
\theta_{T, \alpha}: \operatorname{Tr}_{+}(G) & \rightarrow S^{1}, \\
t & \mapsto\left\{\begin{array}{cc}
\alpha & \text { if } t \in T, \\
\alpha+\pi & \text { if } t \notin T .
\end{array}\right.
\end{aligned}
$$

Clearly, for all $T$ and $\alpha, \theta_{T, \alpha} \in F\left(\operatorname{Tr}(G), S^{1}\right)$.

Lemma 4.11. If $\theta_{T, \alpha} \in F_{\geq}\left(\operatorname{Tr}(G), S^{1}\right)$, then the set of $\theta_{T, \beta}$ for $\beta \in S^{1}$ is a 1dimensional stratum of $F_{\geq}\left(\operatorname{Tr}(G), S^{1}\right)$. Moreover, all 1-dimensional stratums of $F_{\geq}\left(\operatorname{Tr}(G), S^{1}\right)$ are of this form.

Proof. The first point is obvious. Given $\theta \in F_{\geq}\left(\operatorname{Tr}(G), S^{1}\right)$, the orbit $\mathcal{H} \theta$ is 1dimensional if and only if $\theta$ takes only two values, $\beta$ and $\beta+\pi$ for some $\beta \in S^{1}$. Therefore the stratum is 1-dimensional only in this case. This proves the second point.

A more geometric interpretation is that each extreme point of $\operatorname{Rh}(G)$ corresponds to one way of "flattening" $G$ on the line $\mathbb{Z}$.

4.5. Extreme points when $G$ is periodic. In this subsection we consider a quad-graph $G$ embedded in the torus and its lift $\tilde{G}$ to a periodic graph in $\mathbb{R}^{2}$. We wish to understand the rhombic embeddings of $\tilde{G}$ in $\mathbb{R}^{2}$ which are not necessarily periodic.

The extreme points of the space of rhombic embeddings of $\tilde{G}$ are described by Theorem 4.10 above. Associated to an extreme point $\theta_{T, 0}$ is a map from the vertices of $\tilde{G}$ to $\mathbb{Z}$ with the property that the difference between the values at the endpoints of each edge differ by either 1 or -1 : the difference of the map along an edge $e$ which is the transversal of train-track $t \in T$ is 1 (and -1 if $t \notin T$ ).

Let $\pm t_{1}, \ldots, \pm t_{k}$ be the train-tracks of $G$. Let $\mathcal{D}(G)$ be the finite directed graph of positive intersections.

Theorem 4.12. The extreme points $T$ of $\operatorname{Rh}(\tilde{G})$ can be described as follows. Assign elements of $\{+,-, 0\}$ to vertices of $\mathcal{D}(G)$ so that the subgraph consisting of $\{0,+\}$ has no directed cycle and the subgraph consisting of $\{0,-\}$ has no directed cycle. To each such assignment is associated a collection of extreme points of $\tilde{G}$, where $T$ is the union of the set of train-tracks projecting to +-train-tracks of $\mathcal{D}(G)$ and an 
arbitrary subset of the train-tracks projecting to 0-train-tracks of $\mathcal{D}(G)$. Conversely any extreme point of $\operatorname{Rh}(\tilde{G})$ arises in this fashion.

Proof. The graph $\mathcal{D}(\tilde{G})$ is obtained by replacing each vertex of $\mathcal{D}(G)$ by a copy of $\mathbb{Z}$ and each edge from $v$ to $v^{\prime}$ by the collection of all edges from the copy of $\mathbb{Z}$ at $v$ to that at $v^{\prime}$. To a subset $T$ of vertices of $\mathcal{D}(\tilde{G})$, assign elements of $\{0,+,-\}$ to $\mathcal{D}(G)$ as follows: if every vertex of $\mathcal{D}(\tilde{G})$ over $v \in \mathcal{D}(G)$ is in $T$, assign + to $v$. If no vertex is in $T$, assign -. In the remaining case assign 0 . Then a "+" component of $\mathcal{D}(\tilde{G})$ contains a cycle if and only if a $\{0,+\}$-component of $\mathcal{D}(G)$ contains a cycle, and similarly a "-" component of $\mathcal{D}(\tilde{G})$ contains a cycle if and only if a $\{0,-\}$-component of $\mathcal{D}(G)$ contains a cycle.

This result can be formulated differently (and in a slightly more precise way) in terms of the directions of the train-tracks, which can be either 1 or -1 , in an extreme point of $\operatorname{Rh}(\tilde{G})$. Given two non-intersecting oriented train-tracks $t, t^{\prime}$ on $G$, they bound an annulus; we say that they have the same orientation if the oriented path corresponding to one can be deformed to the oriented path corresponding to the other within this annulus. If $t$ and $t^{\prime}$ have the same orientation and another oriented train-track $t^{\prime \prime}$ intersects both, then it intersects both with the same orientation.

Corollary 4.13. (1) To each extreme point of $\operatorname{Rh}(\tilde{G})$ one can associate a maximal subset $T_{0} \subset \operatorname{Tr}_{+}(G)$ of non-intersecting oriented train-tracks with the same orientation, such that if a train-track $t \in \operatorname{Tr}_{+}(\tilde{G})$ has positive (resp. negative) intersection with any train-track projecting to an element of $T_{0}$, then it is mapped to +1 (resp. - 1 ).

(2) Conversely, given a maximal subset $T_{0} \subset \operatorname{Tr}_{+}(G)$ of non-intersecting oriented train-tracks with the same orientation, and a subset $\tilde{T}_{1} \subset \operatorname{Tr}_{+}(\tilde{G})$ of oriented train-tracks projecting to the elements of $T_{0}$, there is an extreme point of $\operatorname{Rh}(\tilde{G})$, defined by the following map $\epsilon: \operatorname{Tr}_{+}(\tilde{G}) \rightarrow\{ \pm 1\}$ :

- if $t$ projects to an element of $T_{0}, \epsilon(t)=1$ iff $t \in \tilde{T}_{1}$,

- if -t projects to an element of $T_{0}, \epsilon(t)=1$ iff $t \notin \tilde{T}_{1}$,

- otherwise, $\epsilon(t)=1$ iff $t$ has positive intersection with a train-track projecting to an element of $T_{0}$.

(3) Given an extreme point $T$ of $\operatorname{Rh}(\tilde{G})$, it is defined as in (2) by a unique choice of $\left(T_{0}, \tilde{T}_{1}\right)$, unless the corresponding function from $\operatorname{Tr}_{+}(\tilde{G})$ to $\{ \pm 1\}$ is the lift of a function from $\operatorname{Tr}_{+}(G)$ to $\{ \pm 1\}$ - in which case $T$ might be described by more than one couple $\left(T_{0}, \tilde{T}_{1}\right)$, always with $\tilde{T}_{1}$ either maximal or empty. Thus the set of extreme points described by more than one couple $\left(T_{0}, \tilde{T}_{1}\right)$ is finite.

Proof. Suppose that (1) is false. Then there are train-tracks $t_{1}^{+}, t_{1}^{-}, t_{2}^{+}, t_{2}^{-} \in \operatorname{Tr}_{+}(\tilde{G})$ such that:

- $t_{1}^{-}$and $t_{1}^{+}$do not intersect, and have the same orientation.

- $t_{2}^{-}$and $t_{2}^{+}$do not intersect, and have the same orientation.

- Both $t_{1}^{+}$and $t_{1}^{-}$intersect $t_{2}^{+}$and $t_{2}^{-}$.

- For $i \in\{1,2\}, t_{i}^{-}$is mapped to -1 and $t_{i}^{+}$to +1 .

This contradicts the definition of $F_{\geq}\left(\operatorname{Tr}_{+}(\tilde{G}), S^{1}\right)$ (or the statement of Theorem 4.12), so it is impossible, and this shows (1).

The proof of (2) is straightforward; one only needs to check that the function $\epsilon$ is in $F_{\geq}\left(\operatorname{Tr}_{+}(\tilde{G}), S^{1}\right)$. 
To prove (3), note that $T_{0}$ is determined as soon as there are two oriented traintracks in $\operatorname{Tr}_{+}(\tilde{G})$ with different images in $\{ \pm 1\}$. Thus, given an extreme point of $\operatorname{Rh}(\tilde{G})$, the couple $\left(T_{0}, \tilde{T}_{1}\right)$ is uniquely determined unless two non-intersecting train-tracks in $\operatorname{Tr}_{+}(\tilde{G})$ with the same orientation always have the same image. But this is true only for functions from $\operatorname{Tr}_{+}(\tilde{G})$ to $\{ \pm 1\}$ which are lifts of functions from $\operatorname{Tr}_{+}(G)$ to $\{ \pm 1\}$. The result follows.

Example $\left(\mathbb{Z}^{2}\right)$. The graph $\mathcal{D}\left(\mathbb{Z}^{2}\right)$ has 4 vertices, corresponding to $\pm t_{h}$, the left- and right-oriented horizontal train-track and $\pm t_{v}$, the up- and down-oriented vertical train-track, and four edges, $t_{h} \rightarrow t_{v} \rightarrow-t_{h} \rightarrow-t_{v} \rightarrow t_{h}$. The extreme points correspond to one of the following four possible situations:

- all train-tracks projecting to $t_{h}$ have image +1 , and the image of the traintracks projecting to $t_{v}$ are arbitrary,

- all train-tracks projecting to $t_{h}$ have image -1 , and the image of the traintracks projecting to $t_{v}$ are arbitrary,

- all train-tracks projecting to $t_{v}$ have image +1 , and the image of the traintracks projecting to $t_{h}$ are arbitrary,

- all train-tracks projecting to $t_{v}$ have image -1 , and the image of the traintracks projecting to $t_{h}$ are arbitrary.

$T_{0}$ is equal to $\left\{t_{v}\right\}$ in the first case, to $\left\{-t_{v}\right\}$ in the second case, to $\left\{-t_{h}\right\}$ in the third case and to $\left\{t_{h}\right\}$ in the last case.

\section{Periodic RHOMbic Embeddings}

5.1. Existence. For graphs on a torus we have the following analog of Theorem 3.1.

Theorem 5.1. Suppose $G$ is a finite graph embedded on a torus, and each face has degree 4 . Then $G$ has a rhombic embedding on a torus if and only if the following two conditions are satisfied:

(1) Each train-track is a simple closed curve.

(2) The lift of two train-tracks to the universal cover intersect at most once.

Proof. The conditions are clearly necessary. The sufficiency is proved by construction: let $\left(p_{i}, q_{i}\right)$ be the integer homology class of $t_{i}$. Define $u_{i}$ to be the unit vector perpendicular to $\left(p_{i}, q_{i}\right)$. To see that this defines an embedding, it suffices to show that each rhombus has positive area. However if oriented train-tracks $t_{i}$ and $t_{j}$ intersect, with $t_{i}$ crossing $t_{j}$ from right to left, then $p_{i} q_{j}-p_{j} q_{i}>0$ since it counts the algebraic intersection number. This implies that $u_{i} \wedge u_{j}>0$.

5.2. Asymptotic directions. Consider a graph $G$ with faces of degree 4 embedded on a torus, satisfying the conditions of Theorem 5.1. Each train-track $t$ is a simple closed curve on the torus; the homology class of this curve is denoted $[t] \in \mathbb{Z}^{2}$. Two train-tracks of homology classes $\left(p_{1}, q_{1}\right)$ and $\left(p_{2}, q_{2}\right)$ intersect $\left|p_{1} q_{2}-p_{2} q_{1}\right|$ times.

Given a rhombic embedding of $G$ on a torus, the lift of an oriented train-track $t$ to the universal cover $\mathbb{R}^{2}$ is a periodic curve and hence has an asymptotic direction $a(t) \in S^{1}$. It also has an asymptotic vector $v(t)$, which is the difference in $\mathbb{R}^{2}$ between two consecutive lifts of a point of $t$. The asymptotic vector of a train-track 
$t$ is of course the sum of the transverse vectors of all rhombi comprising $t$. That is, we have

$$
v\left(t_{i}\right)=\sum_{j} b_{i, j} u_{j},
$$

where the sum is over all train-tracks, $u_{j}$ is the transversal of oriented train-track $j$, and $b_{i, j}$ is the number of intersections of train-track $j$ with train-track $i: b_{i, j}=$ $p_{i} q_{j}-p_{j} q_{i}$. The asymptotic direction is the unit vector $a\left(t_{i}\right)=v\left(t_{i}\right) /\left\|v\left(t_{i}\right)\right\|$.

5.3. The space of periodic rhombic embeddings. Let $\operatorname{Rh}(G)$ be the space of rhombic embeddings of $G$ on a torus (the flat metric on the torus may be a function of the embedding). The argument of section 4.1 can still be used, but we now have to consider only the finite set of train-tracks on the torus. The proof of Theorem 3.1 - in a simplified form - shows that $\operatorname{Rh}(G)$ is convex when parametrized by the rhombus angles, but it is now finite-dimensional, of dimension $|\operatorname{Tr}(G)|-1$.

The characterization of its extreme points can be done just as in Theorem 4.10 and Lemma 4.11 above. We can still consider $\operatorname{Rh}(G)$ as the quotient of $F_{>}\left(\operatorname{Tr}(G), S^{1}\right)$ by the action of $S^{1}$, and the argument using the action of $\mathcal{H}$ still shows that the 1-dimensional stratums of $F_{\geq}\left(\operatorname{Tr}(G), S^{1}\right)$ correspond to its elements which take only two values $\beta$ and $\beta+\pi$.

In particular $\overline{\operatorname{Rh}(G)}$ is a convex polyhedron of dimension $|\operatorname{Tr}(G)|-1$.

5.4. Canonical embedding. On a torus, there is a "best" rhombic embedding of any given graph; it has a simple geometric characterization but is also obtained as the rhombic embedding maximizing the area.

Theorem 5.2. On $\operatorname{Rh}(G)$ the area function of the torus is a strictly concave function of the rhombus angles. There is a unique point maximizing the area, which is characterized by the fact that the transverse direction of each train-track is orthogonal to its asymptotic direction.

Proof. The area of a rhombus of angle $\theta$ is $\sin \theta$, which is a strictly concave function of $\theta \in(0, \pi)$. This implies that the total area, which is the sum of the areas of the individual rhombi, is concave as a function of the angles. To show strict concavity, it suffices to show strict concavity under any one-parameter perturbation. But any perturbation must change the angle of some rhombus, and the contribution from the area of this rhombus is strictly concave. So the area has at most one critical point on $\operatorname{Rh}(G)$.

Let $R$ be a rhombic embedding of graph $G$. The area of $R$ is

$$
\begin{aligned}
A(R) & =\sum_{1 \leq i \neq j \leq t} b_{i, j} u_{i} \wedge u_{j} \\
& =\sum_{1 \leq i \neq j \leq t} b_{i, j} \sin \left(\theta_{j}-\theta_{i}\right),
\end{aligned}
$$

where $t$ is the number of train-tracks in $G, u_{i}=e^{i \theta_{i}}$ and the sum runs over all train-tracks in $G$, with an orientation chosen for each. As a function of $u_{i}$ it is a constant plus $u_{i} \wedge\left(\sum_{j} b_{i, j} u_{j}\right)=u_{i} \wedge a\left(t_{i}\right)$. In particular since $u_{i}$ is a unit vector this quantity is critical if and only if, for each $i, u_{i}$ is orthogonal to $a\left(t_{i}\right)$. So, if the area does have a critical point on $\operatorname{Rh}(G)$, it is as described in the theorem. 
For each $1 \leq i \leq t$, let $\alpha_{i}$ be the angle of the asymptotic direction of $t_{i}$. We define a vector field $V$ on $\operatorname{Rh}(G)$ by

$$
\forall i \in\{1, \cdots, t\}, d \theta_{i}(V)=\cos \left(\alpha_{i}-\theta_{i}\right) .
$$

Clearly $V$ vanishes exactly at the critical points of the area. In fact, $A$ is increasing along the integral curves of $V$ :

$$
\begin{aligned}
& d A(V)=\sum_{i} d u_{i}(V) \wedge \sum_{j} b_{i, j} u_{j}=\sum_{i}\left(-\cos \left(\theta_{i}-\alpha_{i}\right) J u_{i}\right) \wedge v\left(t_{i}\right), \\
& d A(V)=\sum_{i} \cos \left(\theta_{i}-\alpha_{i}\right)\left\langle u_{i}, v\left(t_{i}\right)\right\rangle=\sum_{i}\left\|v\left(t_{i}\right)\right\| \cos ^{2}\left(\theta_{i}-\alpha_{i}\right) \geq 0 .
\end{aligned}
$$

To prove that $\operatorname{Rh}(G)$ contains a critical point of $V$, it is therefore sufficient to prove that, at any point of $\partial \mathrm{Rh}(G)$ where the area is positive, $V$ points towards the interior of $\operatorname{Rh}(G)$. Let $\theta^{0}=\left(\theta_{i}^{0}\right)_{1 \leq i \leq t} \in \partial \operatorname{Rh}(G)$; the face of $\partial \operatorname{Rh}(G)$ containing $\theta^{0}$ is characterized by a set of equalities of the form $\theta_{i_{p}}=\theta_{j_{p}}$ for different values of $p$, where - without loss of generality - $t_{j_{p}}$ has positive intersection with $t_{i_{p}}$.

For each $1 \leq i \leq t$, let $\alpha_{i}^{0}$ be the angle of the asymptotic direction of $t_{i}$ for $\theta^{0}$. Then $\alpha_{i_{p}}^{0}, \alpha_{j_{p}}^{0} \in\left(\theta_{i_{p}}^{0}-\pi, \theta_{i_{p}}^{0}\right)$ and $\alpha_{j_{p}}^{0}>\alpha_{i_{p}}^{0}$ (because $t_{j}$ has positive intersection with $t_{i}$ and $\left.A>0\right)$. Therefore $\cos \left(\theta_{j_{p}}^{0}-\alpha_{j_{p}}^{0}\right)>\cos \left(\theta_{i_{p}}^{0}-\alpha_{i_{p}}^{0}\right)$, and thus $V$ points towards the interior of $\operatorname{Rh}(G)$.

Corollary 5.3. A rhombic graph on a torus has maximal area if and only if, for each train-track $t$, the transverse direction of $t$ is orthogonal to its asymptotic direction $a(t)$.

\section{Parallelogram embeddings}

A generalization of rhombic embeddings is parallogram embeddings, wherein each face is mapped to a parallelogram. Given a parallelogram embedding, a unique rhombic embedding can be obtained by replacing each edge with the unit length vector in the same direction. Conversely, the set of parallelogram embeddings associated to a given rhombic embedding is obtained simply by replacing each edge in a train-track with a real multiple (a different real multiple for each train-track). Thus the combinatorial and topological behavior of parallelogram embeddings can be understood from the underlying rhombic embedding.

\section{ACKNOWLEDGEMENTS}

The second author thanks Gilbert Levitt for some remarks related to this work.

\section{REFERENCES}

[1] R. J. Duffin, Potential theory on a rhombic lattice. J. Combinatorial Theory 5 (1968) 258-272. MR 38:331

[2] Udo Hertrich-Jeromin, Introduction to Möbius Differential Geometry, volume 300 of London Mathematical Society Lecture Note Series. London Mathematical Society, Cambridge, 2003.

[3] R. Kenyon, The Laplacian and Dirac operators on critical planar graphs Invent. Math. 150 (2002), 409-439.

[4] R. Kenyon, An introduction to the dimer model, Lecture notes of the ICTP, to appear. 
[5] C. Mercat, Discrete Riemann surfaces and the Ising model, Comm. Math. Phys. 218 (2001), 177-216. MR 2002c:82019

[6] C. Mercat, Holomorphie discrète et modèle d'Ising, Ph.D. thesis, Université Louis Pasteur, Strasbourg, France, 1998. http://www-irma.u-strasbg.fr/irma/publications/1998/98014.shtml

Laboratoire de Mathématiques, CNRS UMR 8628, Université Paris-Sud, 91405 Orsay, FRANCE

Laboratoire Emile Picard, UMR CNRS 5580, Institut de Mathématiques, Université Paul Sabatier, 118 route de Narbonne, 31062 Toulouse Cedex 4, France

E-mail address: schlenker@picard.ups-tlse.fr 